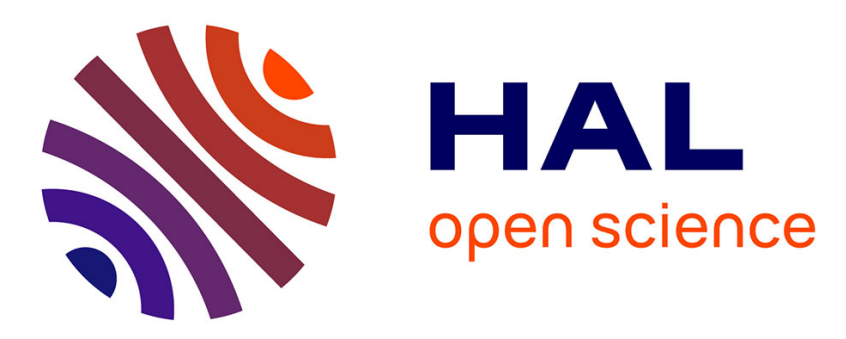

\title{
Metzler Matrix Transform Determination using a Nonsmooth Optimization Technique with an Application to Interval Observers
}

Emmanuel Chambon, Pierre Apkarian, Laurent Burlion

\section{- To cite this version:}

Emmanuel Chambon, Pierre Apkarian, Laurent Burlion. Metzler Matrix Transform Determination using a Nonsmooth Optimization Technique with an Application to Interval Observers . SIAM Conference on Control and its Applications, Jul 2015, Paris, France. hal-01147107

\section{HAL Id: hal-01147107 \\ https://hal.science/hal-01147107}

Submitted on 29 Apr 2015

HAL is a multi-disciplinary open access archive for the deposit and dissemination of scientific research documents, whether they are published or not. The documents may come from teaching and research institutions in France or abroad, or from public or private research centers.
L'archive ouverte pluridisciplinaire $\mathbf{H A L}$, est destinée au dépôt et à la diffusion de documents scientifiques de niveau recherche, publiés ou non, émanant des établissements d'enseignement et de recherche français ou étrangers, des laboratoires publics ou privés. 


\title{
Metzler Matrix Transform Determination using a Nonsmooth Optimization Technique with an Application to Interval Observers*
}

\author{
Emmanuel Chambon ${ }^{\dagger, \ddagger} \quad$ Pierre Apkarian $^{\dagger} \quad$ Laurent Burlion ${ }^{\dagger}$
}

\begin{abstract}
The paper deals with the design of cooperative systems which formulates as computing a state coordinate transform such that the resulting dynamics are both stable and cooperative. The design of cooperative systems is a key problem to determine interval observers. Solutions are provided in the literature to transform any system into a cooperative system. A novel approach is proposed which reformulates into a stabilization problem. A solution is found using nonsmooth optimization techniques.
\end{abstract}

\section{Introduction.}

Cooperative systems - that is systems whose Jacobian matrix is Metzler (see Definition 2.1) - have the interesting property to keep partial ordering between two trajectories [15, 10]. Such a property makes them key candidates for use as interval observers where the goal is to enclose a given variable $x(t)$ between two other timedependent variables $\underline{x}(t) \leq x(t) \leq \bar{x}(t)$ especially when information on any external disturbance is not available. Related works include for example [9]. Many systems including the considered generic launch vehicle model are however not cooperative. A solution to this problem was proposed in [13] where a time-varying change of coordinates is used to define interval observers applied to linear time-invariant non-cooperative systems. It was indeed shown in [12] that in some cases no timeinvariant change of coordinates can be found to guarantee exponential stability of the obtained interval observer. Despite its theoretical interest and the guarantees it offers, this approach may be hard to implement in practice.

More recent works like [14] and [6] interestingly propose techniques to find a static state coordinate transform $P$ and an observer gain $L$ so that $M=$ $P(A-L C) P^{-1}$ is Metzler even for a non-Metzler matrix $A$. Methods based on the numerical resolution of a Sylvester equation were proposed. In these methods

\footnotetext{
${ }^{*}$ This article contains figures in color for better understanding. †Onera - The French Aerospace Lab, BP74025, 2 avenue Édouard Belin, FR-31055 Toulouse Cedex 4, France.

$\ddagger$ Corresponding author: Emmanuel. Chambon@onera.fr.
}

a target matrix $M$ must be provided which may reduce the set of acceptable solutions. A similar approach is used in [4] with the aim to overcome the mentioned limitation of time-invariant change of coordinates. A specific interval observer structure making use of classical observers is developed with an application to nonlinear systems affine in the unmeasured part of the state variables. It is however assumed that the static matrix transform is known. In this paper an alternative technique to these methods is proposed. A Metzler Matrix Transform synthesis method is developed where the computation of both $P$ and $L$ is performed simultaneously which results in $M$ not being fixed a priori.

As far as control theory is concerned, nonsmooth $H_{\infty}$ synthesis optimization techniques as presented in $[3,2]$ offer the possibility to tune a structured controller against multiple control requirements. Recent advances [1] also make it possible to take multiple models into account when robustness is at stake. Admissible requirements include closed-loop poles location or noise rejection. In this article, this control approach will be used to specify estimation quality requirements on gain $L$ which will be used within a Luenberger observer. It is also shown that the problem of finding a pair $(P, L)$ such that $M=P(A-L C) P^{-1}$ is Hurwitz Metzler may be expressed as a control problem on which these techniques will be used.

Computing a Metzler Matrix Transform is difficult. After reformulation into a control problem so as to use nonsmooth $H_{\infty}$ synthesis techniques, a numerical solution to this mathematical problem is proposed. Definitions and notations are recalled in $\S 2$. The paper is structured as follows. In $\S 3$ (resp. $§ 4$ ) the considered mathematical (resp. control) problem is exposed. A solution to these problems is then proposed in $\S 5$. The theory of interval observers and how they can be used to deterministically bracket an estimation error is presented in $\S 6$. Examples of applications are detailed in $\S 7$. In particular, the algorithm and interval observer approach are tested on a generic launch vehicle rigid model in $\S 7.3$. 


\section{Definitions and notations.}

The Laplace transform is denoted $s$. Given two integers $(i, j)$ symbol $\delta_{i j}$ is defined by

$$
\forall(i, j), \delta_{i j}= \begin{cases}1 & \text { if } i=j \\ 0 & \text { otherwise }\end{cases}
$$

Unless mentioned, $i$ and $j$ are integer variables satisfying $1 \leq i, j \leq n$ where $n$ refers to a matrix dimension. $I$ stands for identity matrix with adequate dimension. For a given matrix $A \in \mathbb{R}^{n \times m}$, let denote $A^{+}=\max (A, 0)$ and $A^{-}=A^{+}-A$ where "max" is the element-wise operator.

Definition 2.1. Let $A=\left(a_{i j}\right) \in \mathbb{R}^{n \times n}$. The matrix $A$ is said to be Metzler if

$$
\forall i \neq j, a_{i j} \geq 0
$$

The matrix $A$ is Hurwitz if all its eigenvalues real parts are strictly negative.

Definition 2.2. For given matrices $A$ and $C$, a Metzler Matrix Transform is a pair $(P, L)$ with $P \in \mathbb{R}^{n \times n}$ and $L \in \mathbb{R}^{n \times m}$ s.t. $P(A-L C) P^{-1}$ is Metzler.

\section{Problem statement.}

Let $G=(A, B, C, D)$ a system with $n$ states, $m \leq$ $n$ measurements. It is supposed $(A, C)$ is observable. The following problem is considered for which an optimization-based solution is proposed in $\S 5$.

Problem 3.1. Find a Metzler Matrix Transform $(P, L)$ such that $M=P(A-L C) P^{-1}$ is Hurwitz Metzler.

In this approach $L$ is used to place poles so that a Hurwitz matrix is obtained. It can be identified to an observer gain. In resulting new coordinates, $G$ reformulates into $\mathcal{G}=\left(M, P B, C P^{-1}, D\right)$.

\section{Control problem (application specific).}

When system $G$ or $\mathcal{G}$ expressed in new coordinates should be observed or stabilized, this becomes a control problem. Using nonsmooth $H_{\infty}$ control formalism, this problem can be formulated as follows

Problem 4.1. Let $\mathcal{C}(s, p)$ be a collection of LTI systems depending on tunable parameters $p$. Let $n_{\text {soft }}$ and $n_{\text {hard }}$ two positive integers and $w$ and $z$ two vectors of $\mathbb{R}^{n_{\text {soft }}+n_{\text {hard }}}$. For a given integer $i \leq n_{\text {soft }}+n_{\text {hard }}$, the transfer functions between input $w_{i}$ and output $z_{i}$ over the collection $\mathcal{C}$ is denoted $T_{w_{i} \rightarrow z_{i}}(\mathcal{C}(s, p))$. The problem is to find $p$ satisfying

$$
\min _{p} \max _{i=1, \ldots, n_{\text {soft }}}\left\{\left\|T_{w_{i} \rightarrow z_{i}}(\mathcal{C}(s, p))\right\|_{2 / \infty}\right\}
$$

subject to $\left\|T_{w_{j} \rightarrow z_{j}}(\mathcal{C}(s, p)) \quad\right\|_{2 / \infty} \leq 1$ with $j=$ $1, \ldots, n_{\text {hard }}$

To solve this control problem optimization-based techniques can be used like nonsmooth $H_{\infty}$ synthesis as presented in [1]. These techniques were implemented into numerical solvers as presented in [8]. More specifically the systune routine from the MatlaB(C) Robust Control Toolbox 2012b and higher can be used. An optimal solution is found when the algorithm manages to drive hard constraints below unity while minimizing soft constraints (also called objectives). In $\S 5$ it will be shown that problem 3.1 reformulates into an optimization problem which can be solved using these optimization-based techniques.

\section{Proposed solution.}

Before going into the details of the method, the reader should be reminded that the proposed solution is:

- a numerical solution: it is obtained using an optimization algorithm;

- A local optimal solution: because the problem is non-convex in $(P, L)$ algorithm converges towards an optimal local solution.

Multiple random restarts of the optimization algorithm can be used to find an optimal solution on a larger set of solutions. In case some targeted properties of the solution are not satisfied, it may be necessary to weaken some of the formulated constraints. It may help the optimization algorithm to find a better local solution.

5.1 Note on the existence of a trivial solution. Since diagonal matrices comply with definition 2.1 there exists a trivial solution to problem 3.1.

Proof. With $(A, C)$ observable, choose $L$ so that $A-L C$ eigenvalues are negative real numbers and choose $P$ as the matrix of associated eigenvectors. Then $M=$ $P(A-L C) P^{-1}$ is diagonal Hurwitz hence is a solution to problem 3.1 .

Note this trivial solution may not satisfy control requirements stated in problem 4.1. Also this may lead to large gains $L$ when using pole placement methods.

5.2 Note on Sylvester equation approach. To find Metzler Matrix Transform $(P, L)$ such that $M=$ 
$P(A-L C) P^{-1}$ is Metzler, one can solve the following Sylvester equation as suggested in [14]

$$
P A-M P=Q C
$$

where $A$ is known, $L$ has been obtained solving a pole placement problem and $(M, Q=P L)$ are arbitrarily chosen such that $M$ and $A-L C$ have the same eigenvalues and $M$ is Hurwitz Metzler. Note however that this equation has a unique solution if and only if $A$ and $M$ have distinct eigenvalues. Because the approach presented in the sequel does not rely on an a priori choice of $M$ or $Q$ it offers more freedom in the resolution. On the other hand, the contingency of a local vs. global optimization technique has to be accepted.

5.3 Metzler conditions stabilization problem. Let $M=P(A-L C) P^{-1} \in \mathbb{R}^{n \times n}$ where $P$ and $L$ are decision variables. For $M$ to be Metzler, the $n(n-1)$ following inequalities must be satisfied

$$
\begin{aligned}
\forall i \neq j, M_{i j}=[P & \left.(A-L C) P^{-1}\right]_{i j} \\
& =d_{i}^{\top} P(A-L C) P^{-1} d_{j} \geq 0
\end{aligned}
$$

where $d_{i}=\left(\delta_{i k}\right)_{1 \leq k \leq n}$ is a column vector. For a given pair $(i, j)$, with $i \not \equiv j$, the corresponding inequality can be seen as an "anti-stabilization" problem in the decision variables $P$ and $L$.

5.4 Control synthesis approach and models. To find a pair $(P, L)$ satisfying both $M=P(A-L C) P^{-1}$ and control constraints expressed on $G$ (eventually considered in-line with other systems), any nonsmooth nonconvex optimization technique can be used. It is proposed to use the approach presented in [1] which can handle multiple models to synthesize control laws according to specified constraints. As far as the conditions in $\S 5.3$ are considered, the constraints can be reformulated into the proposed control framework. The following fictitious ${ }^{1}$ systems are considered

$$
\forall i \neq j, G_{M}^{i j}=\left[\begin{array}{c|c}
-M_{i j}(P, L) \in \mathbb{R} & 0_{1 \times 0} \\
\hline 0_{1 \times n} & 0_{1 \times 0}
\end{array}\right]
$$

From a control point of view, ensuring $\forall i \neq$ $j, M_{i j} \geq 0$ is equivalent to stabilizing these systems $G_{M}^{i j}$. The following collections are considered in the control synthesis

\footnotetext{
${ }^{1}$ In the sense they have no input nor output.
}

(C1) $n(n-1)$ unidimensional fictitious systems $\left\{G_{M}^{i j}\right\}_{i, j}$ on which a requirement on closed-loop poles location is expressed (minimum decay and max frequency);

(C2) (optional) $n(n-1)$ unidimensional fictitious systems $\left\{\bar{G}_{M}^{i j}\right\}_{i, j}$ with state matrix $M_{i j}(P, L)-M_{i j}^{\max }$. This helps to restrict the set of acceptable solutions when $0<M_{i j}^{\max }<+\infty$. As such, upon multiple restarts, the optimization algorithm converges more easily due to limitation of the initialising variables excursion around a smaller set of potential local optima;

(C3) Original plant model $G=(A, B, C, D)$ in-line with additional systems eventually depending on $L$ and additional variables to enforce system stabilization or estimation criteria;

(C4) (optional) any other model to enforce specific properties.

\section{Application to Interval Observers.}

The algorithm was developed towards a specific application to interval observers which is presented here. The theory presented in this section is inspired from the works in [13] and [14]. Since the proposed algorithm also synthesizes an observer gain $L$, it is proposed to bracket the estimation error using interval observers so as to frame the plant state $x$ in return.

6.1 Plant model. The following plant model expressed in the state space is considered. Using PopovBelevitch-Hautus Lemma, observability of $(A, C)$ can be checked to account for possibly unobservable eigenvalues.

$$
(G)\left\{\begin{aligned}
\dot{x} & =A x+B_{d} d+B_{u} u \\
& =A x+B\left(\begin{array}{l}
d \\
u
\end{array}\right) \\
y & =C x
\end{aligned}\right.
$$

where $x \in \mathbb{R}^{n}$ and $y \in \mathbb{R}^{m}$ with $m \leq n$. Initial conditions are given by a vector $x_{0}$. Input $d$ is an unknown disturbance but it is supposed there exists known bounds $\underline{d}$ and $\bar{d}$ such that $\forall t, \underline{d}(t) \leq d(t) \leq \bar{d}(t)$. It is supposed a dynamic controller $K(s)$ has been designed ensuring system stability in these conditions. Stabilizing output-feedback control law $u=K(s) y$ is then applied to the system.

6.2 Luenberger observer. The following Luenberger observer is considered 


$$
\left(G_{\text {obs }}\right)\left\{\begin{array}{l}
\dot{\widehat{x}}=A \widehat{x}+B_{u} u+L(y-C \widehat{x}) \\
\widehat{y}=C \widehat{x} \\
\widehat{x}(0)=\widehat{x}_{0}
\end{array}\right.
$$

The objective is to ensure minimal estimation error.

6.3 Estimation error system Let $e=x-\widehat{x}$ the estimation error, it is inferred

$$
\dot{e}=(A-L C) e+B_{d} d
$$

with $e(0)=e_{0}=x_{0}-\widehat{x}_{0}$. The objective is to find $\left(\underline{e}, \bar{e}, \underline{e}_{0}, \bar{e}_{0}\right)$ s.t. for $e_{0} \in\left[\underline{e}_{0}, \bar{e}_{0}\right]$ then $\forall t, \underline{e}(t) \leq e(t) \leq$ $\bar{e}(t)$. The pair $\left(\underline{e}_{0}, \bar{e}_{0}\right)$ is supposed known from now on.

6.4 Interval observer. To use the results presented in [14], a Metzler Matrix Transform needs to be found and the error estimation system expressed into the corresponding new coordinates. It is supposed synthesis in $\S 5$ has returned $(P, L)$ s.t. $M=P(A-L C) P^{-1}$ is Hurwitz Metzler and estimation quality requirements on system (6.9) are satisfied. Using the change of coordinates $e_{z}=P e$, system (6.9) becomes

$$
\begin{aligned}
\dot{e}_{z} & =P(A-L C) P^{-1} e_{z}+P B_{d} d \\
& =M e_{z}+B_{d}^{\prime} d
\end{aligned}
$$

where $B_{d}^{\prime}=P B_{d}$. Using [5, Lemma 1] and notations in $\S 2$, an interval observer for system (6.10) is given by

$$
\left\{\begin{array}{l}
\dot{\dot{e}}_{z}=M \underline{e}_{z}+B_{d}^{\prime+} \underline{d}-B_{d}^{\prime-} \bar{d} \\
\dot{\bar{e}}_{z}=M \bar{e}_{z}+B_{d}^{\prime+} \bar{d}-B_{d}^{\prime-} \underline{d}
\end{array}\right.
$$

under initial conditions

$$
\begin{aligned}
& \underline{e}_{z}(0)=P^{+} \underline{e}_{0}-P^{-} \bar{e}_{0} \\
& \bar{e}_{z}(0)=P^{+} \bar{e}_{0}-P^{-} \underline{e}_{0}
\end{aligned}
$$

This interval observer is composed of two autonomous systems (no dependency on system output $y$ ). Let $T=P^{-1}$, the bounds on $e$ are obtained using [5, Lemma 1]:

$$
\left\{\begin{array}{l}
\underline{e}=T^{+} \underline{e}_{z}-T^{-} \bar{e}_{z} \\
\bar{e}=T^{+} \bar{e}_{z}-T^{-} \underline{e}_{z}
\end{array}\right.
$$

Then for an initial estimation error $e_{0} \in\left[\underline{e}_{0}, \bar{e}_{0}\right]$, a time-varying bracketing of the state $x$ is obtained as $\widehat{x}+\underline{e} \leq x \leq \widehat{x}+\bar{e}$. The system composed of autonomous systems $(6.11)$ with output $y_{e}=(\underline{e}, \bar{e})^{\top}$ is called $\left(G_{e}\right)$.

\section{Examples.}

The following examples are used to demonstrate the possibilities of the algorithm detailed here with an application to interval observers. Note that syntheses were performed using systune function from the Robust Control Toolbox 2014b [11] correctly parametrized to benefit from the Parallel Computing Toolbox. Other implementations may be used.

7.1 Third-order system with unobservable mode. This example is inspired from the partial linear system example presented in [14]. It is given by

$$
A=\left[\begin{array}{rrr}
2 & 0 & 0 \\
1 & -4 & \sqrt{3} \\
-1 & -\sqrt{3} & -4
\end{array}\right], C=\left[\begin{array}{lll}
1 & 0 & 0
\end{array}\right]
$$

The matrix $B$ is not recalled here since no disturbance input is considered. The problem reduces to finding a suitable Metzler Matrix Transform with limited control constraints. Note however that the complex mode $-4 \pm \mathbf{j} \sqrt{3}$ is not observable.

To solve this problem, collections (C1) and (C2) with $n=3$ and $\forall(i, j), M_{i j}^{\max }=10$ are used. Moreover using (C4), $A-L C$ eigenvalues real part are forced to stay within $\left[-10,-3 \cdot 10^{-3}\right]$. A solution is typically found in less than 5 iterations and after 3 restarts. The following results have been computed

$$
\begin{aligned}
& M=\left[\begin{array}{rrr}
-2.0764 & 1.5488 & 0.0611 \\
0.2614 & -2.4897 & 9.8946 \\
0.6941 & 0.0094 & -3.8150
\end{array}\right], \\
& P=\left[\begin{array}{rrr}
1.0991 & -0.6915 & -0.7497 \\
0.7811 & 1.7077 & 0.1462 \\
0.2593 & -0.2680 & 0.2964
\end{array}\right], L=\left[\begin{array}{r}
2.3811 \\
0.3125 \\
-1.2164
\end{array}\right]
\end{aligned}
$$

It is readily verified that $M=P(A-L C) P^{-1}$ is Hurwitz Metzler. According to results in $\S 6$ one can note that the estimation error depends on the initial error but converges towards zero since no other disturbance is considered.

7.2 Sixth-order system with two complex modes. This example is inspired from the one presented in [13]. It is given by

$$
\begin{gathered}
A=\left[\begin{array}{rrrrrr}
-1 & 1 & 0 & 0 & -1 & 0 \\
-1 & -2 & 0 & -1 & 0 & 1 \\
-2 & 0 & -3 & -2 & 0 & 0 \\
-1 & 0 & -2 & -3 & 0 & 1 \\
-1 & 0 & 2 & 0 & -4 & 0 \\
-1 & -1 & 0 & 1 & 0 & -1
\end{array}\right], B=\left[\begin{array}{rr}
0 & -18 \\
0 & -13 \\
0 & -5 \\
0 & -4 \\
0 & -10 \\
0 & 22
\end{array}\right], \\
C=\left[\begin{array}{llllll}
1 & 0 & 0 & 0 & 0 & 0
\end{array}\right], D=\left[\begin{array}{lr}
1 & 0
\end{array}\right]
\end{gathered}
$$


The first input corresponds to a disturbance input on the measurement. Its effect on the estimation quality needs to be mitigated using an appropriate disturbance rejection requirement. Note that since $y=C x+d$, the estimation error system is defined by

$$
\dot{e}=(A-L C) e-L d
$$

rather than by (6.9). To solve this problem, a collection of synthesis models composed of (C1) to (C4) with $n=6$ and $\forall(i, j), M_{i j}^{\max }=1.10^{2}$ is used. Estimation quality is ensured through minimization of the $\mathrm{H}_{2}$ norm of the transfer from $d$ to $e$ using model (C3). Stability of the estimation error subsystem is ensured through (C4) where $A-L C$ eigenvalues real parts are forced to stay in the interval $\left[-10,-3 \cdot 10^{-3}\right]$. A solution is typically found in 897 iterations and after 4 restarts, see (7.18) on Fig. 1. A similar construction to the one presented in $\S 6$ can then be used to frame state $x$.

\subsection{Fifth-order rigid launcher longitudinal} model. The approach in $\S 6$ is applied to a generic rigid launch vehicle longitudinal model with unknown wind input. No uncertainty is considered in this article. Like other examples, the method proposed in $\S 5$ is used to compute $P$ and $L$. Simulations are then run using a simplified wind profile.

7.3.1 Model. The model (7.19) in Fig. 2 is considered where the first input corresponds to the unknown wind input $d$ and $D=0$. The second input corresponds to the thruster orientation input signal to which the stabilizing control law is applied. Note that $n=5$.

7.3.2 Synthesis and results. To solve the problem, the collections of models (C1) to (C4) are used where $\forall(i, j), M_{i j}^{\max }=50$ and $A-L C$ eigenvalues real parts are forced to lie in $\left[-1.10^{2},-1.10^{-2}\right]$. Using model (C3) a minimization constraint on the weighted $\mathrm{H}_{2}$ norm from unknown input $d \in \mathbb{R}$ to estimation error $e \in \mathbb{R}^{5}$ is expressed. The optimization algorithm generally gives a solution in around 1100 iterations and 6 restarts. Results are shown in (7.20) in Fig. 2.

7.3.3 Simulation. Since the system is unstable, the simulation is performed after structured $H_{\infty}$ synthesis of a dynamic stabilizing controller $K(s)$ for this system. As this is not the main purpose of this work, it is not detailed here. Fig. 3 illustrates how the different systems $G, G_{\mathrm{obs}}$ and $G_{e}$ are related. Note that system $G_{e}$ does not interact with the stabilized plant model. Using wind profile shown in Fig. 4 with $+/-7 \mathrm{~m} / \mathrm{s}$ uncertainty on wind speed, the results shown in Fig. 5
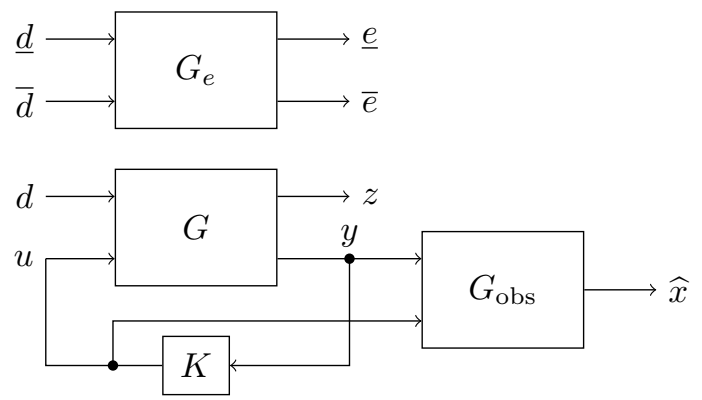

Figure 3: Simulation model where $\left(G_{e}\right)$ is a combination of two autonomous systems.

and 6 are obtained. As expected it is interesting to note that despite state estimate is used to express boundaries on the state, it does not necessarily lie in the resulting interval $[\widehat{x}+\underline{e}, \widehat{x}+\bar{e}]$ since $\underline{e}$ (resp. $\bar{e}$ ) can be positive (resp. negative).

7.4 Execution summary. Executions were performed running MatlaB(C) R2014b with Parallel Computing Toolbox version 6.5 on a Windows@ $7-64$ bits station with 8 Go RAM and Intel(c) Xeon processor E5$1607 \mathrm{v} 2-3 \mathrm{GHz}$ (quad-core). A summary of these executions for each example is shown in Table 1. Note the number of iterations and time results are only indicative since they may vary from one run to another but they are representative of the complexity of the problem.

\section{Conclusions.}

In this article an application of nonsmooth optimization techniques [1] to numerically solve a mathematical problem combined to a control problem has been pre-

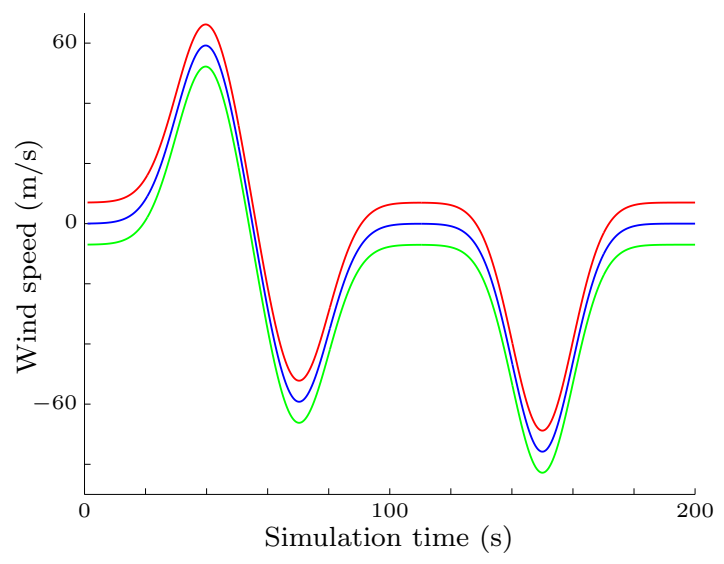

Figure 4: Wind speed profile (in blue) and known bounds $\underline{d}$ and $\bar{d}$ used in simulation. 


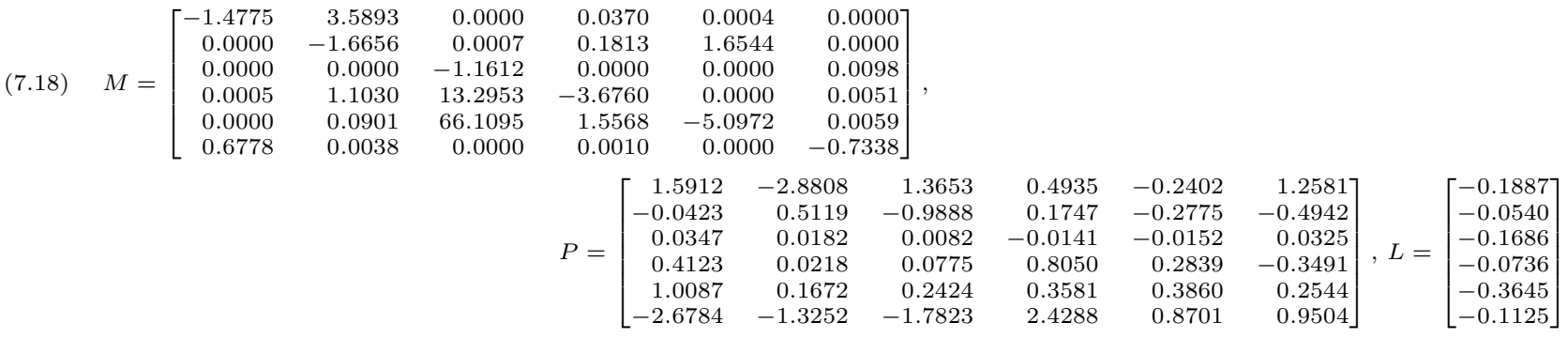

Figure 1: A solution obtained on example (7.16).

(7.19)

\begin{tabular}{|c|c|c|c|c|c|c|c|c|c|c|c|c|c|c|}
\hline \multirow{5}{*}{$A=$} & 0 & 1 & 0 & 0 & $0^{-}$ & & 0 & $0^{-}$ & & & & & & \\
\hline & -893.1828 & -40.1197 & 0 & 878.6575 & 0 & & 0 & 0 & & $\lceil 0$ & 0 & 0 & 0 & $1]$ \\
\hline & 0 & 0 & 0 & 1 & 0 &,$B=$ & 0 & 0 &,$C=$ & 0 & 0 & 1 & 0 & 0 \\
\hline & 0 & 0 & 1.4821 & 0 & 0.0026 & & -0.0024 & -3.6490 & & 1 & 0 & 0 & 0 & 0 \\
\hline & 0 & 0 & -42.8326 & 0 & -0.0294 & & 0.0296 & -22.3667 & & & & & & \\
\hline
\end{tabular}

$(7.20) \quad M=\left[\begin{array}{rrrrr}-22.4368 & 0.4289 & 0.0045 & 0.0156 & 0.6777 \\ 0.0147 & -14.0849 & 0.0075 & 0.0041 & 0.0551 \\ 16.4291 & 49.9966 & -30.1315 & 7.9257 & 21.0740 \\ 2.7674 & 2.5858 & 0.0401 & -25.4104 & 0.0992 \\ 2.1787 & 18.5450 & 0.0935 & 13.6873 & -28.7126\end{array}\right]$,

$$
P=\left[\begin{array}{rrrrr}
46.2716 & 2.6470 & 11.3937 & -103.5197 & -8.1488 \\
-14.4955 & -0.5588 & -3.7667 & 34.9205 & 2.8661 \\
63.4497 & 4.6807 & 462.9193 & -164.6066 & -119.5850 \\
27.7039 & 1.4743 & -1.7876 & -58.8815 & -3.4144 \\
10.3172 & -0.5000 & -10.8160 & 1.7723 & 0.8932
\end{array}\right], L=\left[\begin{array}{rrr}
-0.7252 & 3.8941 & 23.5926 \\
-71.0519 & 87.4368 & -543.8054 \\
0.3297 & 24.8749 & 0.9810 \\
-2.8577 & 7.6596 & 9.1507 \\
32.1596 & -68.2297 & 5.3586
\end{array}\right]
$$

Figure 2: Rigid launch vehicle longitudinal model with detectors dynamics (top, see §7.3) and obtained Metzler Matrix Transform (bottom).

\begin{tabular}{|c|c|c|c|c|r|r|c|}
\hline Ex. & Res. & Dim. $(n)$ & \# Models & Restarts & Iter. & Time (s) & Ref. \\
\hline$(7.14)$ & $(7.15)$ & 3 & 13 & 3 & 1 & 16 & {$[14]$} \\
$(7.16)$ & $(7.18)$ & 6 & 61 & 4 & 897 & 513 & {$[13]$} \\
$(7.19)$ & $(7.20)$ & 5 & 41 & 6 & 1161 & 115 & \\
\hline
\end{tabular}

Table 1: Metzler Matrix Transform synthesis typical execution summary.

sented. To determine a Metzler Matrix Transform the corresponding mathematical problem 3.1 was reformulated into a control problem 4.1. Combined with control requirements, a local optimal solution to this problem can be found. This approach has been successfully applied to various examples including a rigid launch vehicle model. In comparison with other methods, this approach allows for simultaneous tuning of $P$ and $L$ and does not make assumption on the targeted $M$ matrix structure. Moreover, by benefiting from observer structures as proposed in [14] and [4] implementing the resulting interval observer is straightforward in practise.

Further improvements will be dedicated to reduce complexity since non-negligible computing resources are needed with multiple algorithm restarts before converging towards an acceptable solution especially in the case of higher-dimensional problems. Other problems could also be considered like finding static $P$ such that $P[A(y)-L(y) C] P^{-1}$ is Metzler where $y \in \mathbb{R}^{m}$ is the known system output signal. The interest would be to benefit from the interval observer structure proposed in [4]. In this work knowing such $P$ is considered an hy- 

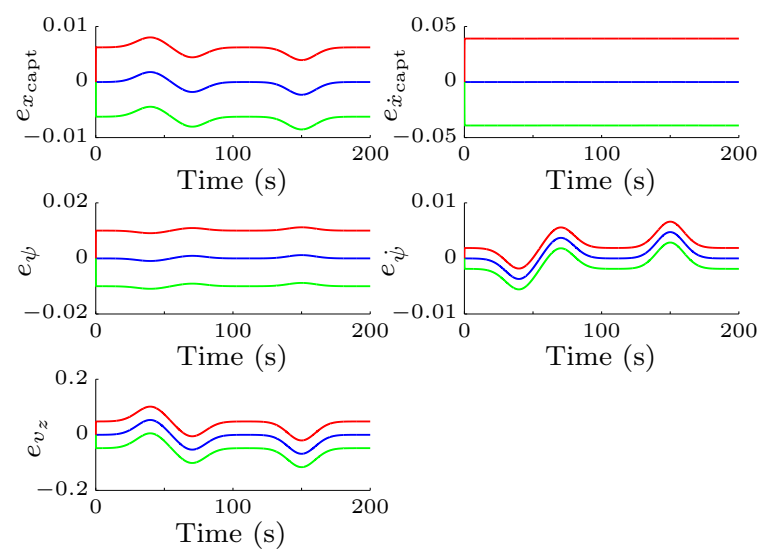

Figure 5: Simulation results on example (7.19): estimation error $e=x-\widehat{x}$ is represented in blue. The bounds obtained using interval observer expression as in (6.11) are represented in red and green.
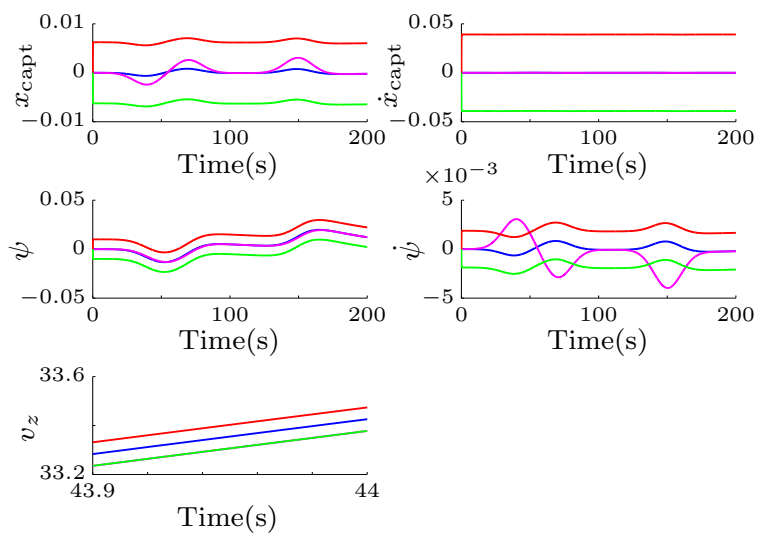

Figure 6: Simulation results on example (7.19) using wind profile in Fig. 4: the state (blue), the state estimate (magenta) and the upper (resp. lower) bound (red, resp. green) are represented. Zoom is performed on otherwise indistinguishable curves.

pothesis, which might be limiting. Also note that other systems like positive continuous-time linear systems [7] also have a Metzler state-space matrix which gives hints on a wider scope for this article.

\section{References}

[1] P. ApKarian, Tuning controllers against multiple design requirements, in Proc. of the American Control Conference, Washington, USA, June 2013, pp. 38883893.
[2] P. ApKARIAN AND D. Noll, Nonsmooth $H_{\infty}$ synthesis, IEEE Transactions on Automatic Control, 51 (2006), pp. 71-86.

[3] J. V. Burke, D. Henrion, A. S. Lewis, and M. L. OverTon, HIFOO - a MATLAB package for fixedorder controller design and $H_{\infty}$ optimization, in Proc. of the 5th IFAC Symposium on Robust Control Design, Toulouse, France, Aug. 2006.

[4] T. N. Dinh, F. Mazenc, And S.-I. Niculescu, Interval observer composed of observers for nonlinear systems, in Proc. of the European Control Conference, Strasbourg, France, June 2014, pp. 660-665.

[5] D. Efimov, T. Raïssi, S. Chebotarev, and A. ZolGHADRI, Interval state observer for nonlinear timevarying systems, Automatica, 49 (2013), pp. 200-205.

[6] D. Efimov, T. Raïssi, And A. Zolghadri, Control of nonlinear and LPV systems: Interval observer-based framework, IEEE Transactions on Automatic Control, 58 (2013), pp. 773-778.

[7] L. Farina And S. Rinaldi, Positive Linear Systems: Theory and Applications, John Wiley \& Sons, 2000.

[8] P. Gahinet And P. Apkarian, Frequency-domain tuning of fixed-structure control systems, in Proc. of the UKACC International Conference on Control, Sept. 2012, pp. 178-183.

[9] J. L. Gouzé, A. Rapaport, And M. Z. HADJSADOK, Interval observers for uncertain biological systems, Ecological Modelling, 133 (2000), pp. 45-56.

[10] L. MAILlERET, Stabilisation globale des systèmes positifs mal connus - Applications en Biologie, PhD thesis, Université de Nice Sophia-Antipolis, Nice, May 2004.

[11] MATLAB, Robust Control Toolbox version 5.2 (R2014b), The MathWorks Inc., Natick, Massachusetts, 2014.

[12] F. MAZEnC And O. Bernard, Asymptotically stable interval observers for planar systems with complex poles, IEEE Transactions on Automatic Control, 55 (2010), pp. 523-527.

[13] _, Interval observers for linear time-invariant systems with disturbances, Automatica, 47 (2011), pp. 140-147.

[14] T. RaÏssi, D. Efimov, AND A. Zolghadri, Interval state estimation for a class of nonlinear systems, IEEE Transactions on Automatic Control, 57 (2012), pp. 260-265.

[15] H. L. SмIтh, Monotone dynamical systems: an introduction to the theory of competitive and cooperative systems, Bulletin of the American Mathematical Society, 33 (1996), pp. 203-209. 